\title{
TWO MAPPING PROPERTIES OF SCHLICHT FUNCTIONS
}

\section{J. L. ULLMAN}

The mapping properties we shall prove hold for the normalized exterior mapping function of a simple analytic curve. Let $C$ be a simple analytic curve in the $z$-plane and designate its exterior by $D$. The normalized exterior mapping function of $C$ is the analytic function $w=f(z)$ which is uniquely determined by the conditions that (i) it is regular in $D$ except for a simple pole at $z=\infty$, (ii) its power series expansion about $z=\infty$ has the normalization

$$
w=z+a_{0}+\frac{a_{1}}{z}+\cdots,
$$

and (iii) it maps $D$ in a 1-1 manner onto the exterior of a circle $\Sigma$, $|w|=\rho$.

THEOREM I. Let $C$ be a simple analytic curve, and designate its exterior by $D$. Let $f(z)$ be the normalized exterior mapping function of $C$. Let $\sigma$ be a circle with center $z_{0}$, whose closed interior lies in $D$. Then $F(z)$ $=f(z) /\left(z-z_{0}\right)$ maps $\sigma$ onto a curve in the w-plane that is star-shaped from the point $w=0$.

Proof. A curve $\Gamma$ is star-shaped with respect to a point $A$ in its interior if it is a simple curve, and if each point of $\Gamma$ can be connected to $A$ by a straight line lying in the interior of $\Gamma$. Let $\sigma$ have radius $r$, and let $Z$ be a point on $\sigma$. Then $Z-z_{0}=r e^{i \theta}$. Let $F(Z)=R e^{i \phi}$. For the image of $\sigma$ to be star-shaped, $d \phi / d \theta$ must not vanish, and be of constant sign for $0 \leqq \theta<2 \pi$. Since $F(z)$ has a simple pole in $\sigma$, and otherwise is regular and nonzero there, $\phi$ decreases by $2 \pi$ when $\theta$ increases by $2 \pi$, so $d \phi / d \theta$ must be negative for some value $\theta^{\prime}, 0 \leqq \theta^{\prime}<2 \pi$. We now show that it is negative for each value of $\theta$ in the interval.

We first express $d \phi / d \theta$ at a point $Z$ on $\sigma$ in terms of $f(Z)$. Start with

$$
\begin{aligned}
\frac{d \phi}{d \theta} & =\frac{d}{d \theta} \operatorname{Im} \log F(Z) \\
& =\frac{d}{d \theta} \operatorname{Im}\left(\left(\log f(Z)-\log \left(Z-z_{0}\right)\right) .\right.
\end{aligned}
$$

Presented to the Society, November 26, 1949; received by the editors May 26, 1950 and, in revised form, July 24, 1950. 
Differentiate, to obtain

$$
\frac{d \phi}{d \theta}=\operatorname{Im}\left(\frac{f^{\prime}(Z)}{f(Z)} \frac{d Z}{d \theta}-\frac{1}{Z-z_{0}} \frac{d Z}{d \theta}\right) .
$$

Substituting $d Z / d \theta=i\left(Z-z_{0}\right)$, we obtain

$$
\begin{aligned}
\frac{d \phi}{d \theta} & =\operatorname{Im}\left(i\left(Z-z_{0}\right) \frac{f^{\prime}(Z)}{f(Z)}-i\right) \\
& =\operatorname{Re}\left(\left(Z-z_{0}\right) \frac{f^{\prime}(Z)}{f(Z)}-1\right) .
\end{aligned}
$$

We now use the Cauchy integral formula to obtain a representation for $f^{\prime}(Z) / f(Z)$. Since $f^{\prime}(z) / f(z)$ is regular in $D$, and tends to zero as $z \rightarrow \infty$, and since each point $Z$ lies in $D$, for a fixed $Z$ we have

$$
\frac{f^{\prime}(Z)}{f(Z)}=\frac{1}{2 \pi i} \int_{C^{-}} \frac{1}{z-Z} \frac{f^{\prime}(z)}{f(z)} d z .
$$

Let $f(z)=\rho e^{i \alpha}$ when $z$ is on $C$, and indicate the inverse of $w=f(z)$ by $z=z(w)$. Then $\left(f^{\prime}(z) / i f(z)\right) d z=d \alpha$ and from (5) we have

$$
\frac{f^{\prime}(Z)}{f(Z)}=\frac{1}{2 \pi} \int_{0}^{2 \pi} \frac{1}{Z-z\left(\rho e^{i \alpha}\right)} d \alpha .
$$

Substituting (6) in (4), we obtain

$$
\frac{d \phi}{d \theta}=\operatorname{Re}\left(\frac{1}{2 \pi} \int_{0}^{2 \pi} \frac{Z-z_{0}}{Z-z\left(\rho e^{i \alpha}\right)} d \alpha-1\right) .
$$

Since $(1 / 2 \pi) \int_{0}^{2 \pi} d \alpha=1$, this can be written

$$
\begin{aligned}
\frac{d \phi}{d \theta} & =\operatorname{Re}\left(\frac{1}{2 \pi} \int_{0}^{2 \pi}\left(\frac{Z-z_{0}}{Z-z\left(\rho e^{i \alpha}\right)}-1\right) d \alpha\right) \\
& =\frac{1}{2 \pi} \int_{0}^{2 \pi} \operatorname{Re}\left(\frac{z\left(\rho e^{i \alpha}\right)-z_{0}}{Z-z\left(\rho e^{i \alpha}\right)}\right) d \alpha .
\end{aligned}
$$

The integrand in (8) is a continuous function of $\alpha$ since the circumference of $\sigma$ is bounded from $C$. Hence, to prove $d \phi / d \theta<0$, it suffices to show that the integrand in (8) is negative for $\alpha, 0 \leqq \alpha<2 \pi$. Indeed, let $\alpha_{1}$ be a value in this interval, and let $z\left(\rho^{i \alpha_{1}}\right)=z_{1}$. Then

$$
\operatorname{Re} \frac{z_{1}-z_{0}}{Z-z_{1}}<0
$$

if 


$$
\operatorname{Re} \frac{Z-z_{1}}{z_{1}-z_{0}}<0
$$

We write

$$
\operatorname{Re} \frac{Z-z_{1}}{z_{1}-z_{0}}=\operatorname{Re} \frac{Z-z_{0}+z_{0}-z_{1}}{z_{1}-z_{0}}=\operatorname{Re} \frac{Z-z_{0}}{z_{1}-z_{0}}-1
$$

Since $\left|Z-z_{0}\right|<\left|z_{1}-z_{0}\right|$, we have

$$
\operatorname{Re} \frac{Z-z_{0}}{z_{1}-z_{0}}-1 \leqq\left|\frac{Z-z_{0}}{z_{1}-z_{0}}\right|-1<0 .
$$

From (12) it follows that the integrand in (8) is negative for each value of $Z$ on $\sigma$, and the theorem is proved.

Theorem II. Let $C$ be a simple analytic curve, and designate its exterior by $D$. Let $f(z)$ be the normalized exterior mapping function of $C$. Let $Z_{1}$ and $Z_{2}$ be two points in D. If $Z_{1}$ and each point of $C$ lie on the same side of the perpendicular bisector, $L$, of the line joining $Z_{1}$ and $Z_{2}$, then $\left|f\left(Z_{1}\right)\right|<\left|f\left(Z_{2}\right)\right|$.

Proof. Representation (6) for $f^{\prime}(Z) / f(Z)$ is valid for $Z$ in $D$, hence by integration

$$
\log f\left(Z_{i}\right)=\frac{1}{2 \pi} \int_{0}^{2 \pi} \log \left(Z_{i}-z\left(\rho e^{i \alpha}\right)\right) d \alpha \quad(i=1,2) .
$$

It then follows that

$$
\log \left|\frac{f\left(Z_{1}\right)}{f\left(Z_{2}\right)}\right|=\frac{1}{2 \pi} \int_{0}^{2 \pi} \log \left|\frac{Z_{1}-z\left(\rho e^{i \alpha}\right)}{Z_{2}-z\left(\rho e^{i \alpha}\right)}\right| d \alpha .
$$

The integrand in (14) is a continuous function of $\alpha$. From the hypothesis it follows that $\left|\left(Z_{1}-z\left(\rho e^{i \alpha}\right)\right) /\left(Z_{2}-z\left(\rho e^{i \alpha}\right)\right)\right| \leqq 1$. The inequality must hold for some $\alpha$, for otherwise $z\left(\rho e^{i \alpha}\right)$ would be restricted to a line and $C$ would not be simple. Hence the integral in (14) is negative and $\left|f\left(Z_{1}\right)\right|<\left|f\left(Z_{2}\right)\right|$. This completes the proof.

Theorem I provides a complement to results stated by PólyaSzegö $\left[1\right.$, pp. 104-105]. ${ }^{1}$ It also gives part of the domain of schlichtness of the ratio of two schlicht functions.

At the suggestion of the referee we shall discuss Theorem II*, which is Theorem II under the more general hypothesis that $D$ is an arbitrary, simply-connected domain containing $z=\infty$, its bound-

1 This refers to the bibliography at the end of the paper. 
ary set $C$ is not an analytic curve (since this case is covered by Theorem II), and $f(z)$ is the analytic function with normalization (1) which maps $D$ in a 1-1 manner onto the exterior of a circle $\Sigma$, $|w|=\rho$. We lose no generality in taking $L$ to be the real axis, in which case $Z_{1}$ and $Z_{2}$ can be replaced by $Z$ and $Z^{*},{ }^{2}$ where $\operatorname{Im} Z>0$. If $C$ is in the half-plane $\operatorname{Im} z>0$, a level curve $C_{1}$ of $w=f(z)$ also lies in this half-plane with $Z$ in its exterior, and this curve can be used in place of $C$ in the proof used for Theorem II. This shows that $|f(Z)|$ $<\left|f\left(Z^{*}\right)\right|$. If $C$ lies in the half-plane $\operatorname{Im} z \geqq 0$, touching $L$, the level curves of $f(z)$ will all be cut by $L$. Select a sequence, $\left\{C_{k}\right\}$, whose exteriors exhaust $D$. In the proof of Theorem II, replace $C$ by $C_{n}$ to obtain

$$
\log \left|\frac{f(Z)}{f\left(Z^{*}\right)}\right|<\epsilon_{n}
$$

where $\epsilon_{n}>0$ and $\epsilon_{n}$ tends to zero as $n$ tends to $\infty$ ! Hence

$$
|f(Z)| \leqq\left|f\left(Z^{*}\right)\right| \text {. }
$$

We now show that equality in (16) implies that $C$ coincides with $L$. Suppose $\left|f\left(Z_{1}\right)\right|=\left|f\left(Z_{1}^{*}\right)\right|$, where $Z_{1}$ is an interior point of $D$, satisfying $\operatorname{Im} Z_{1}>0$. Since (16) holds in a neighborhood of $Z_{1}, f(z)$ must satisfy the functional relationship

$$
f(z)=e^{i \alpha}\left(f\left(z^{*}\right)\right)^{*},
$$

where $\alpha$ is a constant, $0 \leqq \alpha<2 \pi$. Letting $x \rightarrow \infty, z=x+i y$, we see that, because of normalization (1), $\alpha$ must equal 0 . But then, interpreted geometrically, (17), with $\alpha=0$, implies that $C$ is symmetric about $L$. Since $C$ lies in the half-plane $\operatorname{Im} z \geqq 0$, it must coincide with $L$. Since $C$ is the boundary of a simply-connected domain, it is an interval of $L$. Hence

$$
f(z)=\frac{(a z+b)+\left((a z+b)^{2}-1\right)^{1 / 2}}{2 a}
$$

where $a, b$ are real, $a>0$, and the branch is determined by choosing the positive sign of the radical for $z$ positive and sufficiently large.

\section{BIBLIOGRAPHY}

1. G. P6lya and G. Szegö, Aufgaben und Lehrsätze aus der Analysis, vol. 1, New York, Dover, 1945.

\section{UNIVERSITY OF MICHIGAN}

${ }^{2} Z^{*}$ denotes the complex conjugate of $Z$. 